\title{
NEW LUNG ADENOCARCINOMA CLASSIFICATION IN DAILY PRACTICE
}

\author{
Myriam Remmelink \\ Service d'Anatomie Pathologique, CUB-ULB HOPITAL ERASME Bruxelles \\ e-mail: myriam.remmelink@erasme.ulb.ac.be \\ doi:10.5152/tcb.2012.23
}

\begin{abstract}
Although histologic criteria remain the foundation of this new classification, it is based on an integrated multidisciplinary platform and defines pathologic entities. One of the novelties is discontinuing the use of the term bronchioloalveolar adenocarcinoma (BAC). New entities are described: "Adenocarcinoma in situ "(AIS) and "Minimally invasive adenocarcinoma (MIA). These modifications of the classification have implications for surgeons with new surgical strategies in the future. For invasive adenocarcinomas, the proposition described here is to present a comprehensive histologic subtyping: individual tumors are then classified according to the predominant pattern and the percentages of the subtypes are also reported. The other part of the work is the establishment of a classification of non-small cell lung carcinomas (NSCLC) for small biopsies and cytology. Subclassification in small biopsies is more problematic. There is however universal agreement that most NSCLC can be classified on H\&E stained slides alone without using any immunohistochemical stains and tumors that are readily classifiable on H\&E-stained slides should not be stained with immunohistochemical markers because of the obligation for the pathologist to keep tissue for molecular testing. However, some NSCLC are too poorly differentiated to be accurately subclassified by standard H\&E criteria. Some markers, especially when used in combination, are indeed useful and accurate in subclassifying poorly differentiated NSCLC. Strategic use of small biopsy and cytology samples is important in order to preserve as much tissues as possible for potential molecular studies. One way to attain this is for each institution to develop a multidisciplinary team that coordinates the optimal approach to obtaining and processing biopsy/cytology specimens to provide expeditious diagnostic and molecular results. In conclusion, the new lung adenocarcinoma classification will not only modify daily practice but will also improve the management of patient.
\end{abstract}

Key words: Lung, adenocarcinoma, classification, histologic, pathology

Last year in the Journal of Thoracic Oncology, a multidisciplinary group of experts from the International Association for the Study of Lung Cancer (IASLC)/ American Thoracic Society (ATS) and European Respiratory Society (ERS) proposed a new classification of lung adenocarcinoma (1). This work is very important because lung cancer is the most frequent cause of major cancer incidence and mortality worldwide. Adenocarcinoma is the most common histologic subtype of lung cancer, accounting for almost half of all lung cancers. Enormous resources are spent on trials involving molecular and therapeutic aspects, so it is mandatory to use standardized criteria to avoid confusion and difficulties in comparing studies.

One of the major outcomes of this project is the recognition that the diagnosis of lung adenocarci- noma requires a multidisciplinary approach, which is due to remarkable advances in the areas of medical oncology, molecular biology and radiology. Although histologic criteria remain the foundation of this new classification, it is based on an integrated multidisciplinary platform and defines clinically relevant pathologic entities.

The new classification of lung adenocarcinoma is presented in Table 1 (1). One of the novelties is discontinuing the use of the term bronchioloalveolar carcinoma (BAC) because this term is used for a broad spectrum of tumours. The former BAC was divided into the five categories described below. It is suggested that when the new proposed terms are used, it will be accompanied in parentheses by "(formerly BAC)" (Table 2) (1). 
Table 1. IASLC/ATS/ERS Classification of Lung Adenocarcinoma in Resection Specimens

Preinvasive lesions

Atypical adenomatous hyperplasia

Adenocarcinoma in situ

Minimally invasive adenocarcinoma (Nonmucinous, Mucinous, Mixed)

Invasive adenocarcinoma

Lepidic predominant

Acinar predominant

Papillary predominant

Micropapillary predominant

Solid predominant with mucin production

Variants of invasive adenocarcinoma

Invasive mucinous adenocarcinoma

Colloid

Fetal

Enteric

Solitary adenocarcinoma with lepidic growth (growth restricted to neoplastic cells along preexisting alveolar structures) appears as a ground glass nodule (GGN) on chest computed tomography. In the new classification size of the nodule is important. If smaller than $3 \mathrm{~cm}$, the pathologist has to perform an entire histologic sampling of the surgical specimen because of the definition of new categories as "Adenocarcinoma in situ "(AIS) and "Minimally invasive adenocarcinoma (MIA).

Adenocarcinomas in situ (AIS) represent small $(\leq 3 \mathrm{~cm})$ solitary adenocarcinomas with pure lepidic growth. AIS are subdivided into mucinous and nonmucinous variants. Virtually all cases of AIS are nonmucinous, consisting of type II pneumocytes and /or Clara cells. It defines patients who should have $100 \%$ disease-specific survival if the lesion is completely resected. AIS is one of the lesions formerly known as BAC.

"Minimally invasive adenocarcinomas (MIA)" are small $(\leq 3 \mathrm{~cm})$ solitary adenocarcinomas with predominant lepidic growth and small foci of invasion measuring $\leq 0.5 \mathrm{~cm}$. The invasive component to be measured in MIA is defined as follows: (1) histologic subtypes other than a lepidic pattern or (2) tumour cells infiltrating myofibroblastic stroma. MIA is excluded if the tumour (1) invades lymphatics, blood vessels, or pleura or (2) contains tumour necrosis. MIA is one of the lesions formerly known as BAC. It defines patients who have near $100 \%$ disease specific survival, if completely resected. MIA is usually non-mucinous. These
Table 2. Categories of new Adenocarcinoma Classification former Bronchioloalveolar carcinoma

Adenocarcinoma in situ

Minimally invasive adenocarcinoma

Lepidic predominant adenocarcinoma (nonmucinous)

Adenocarcinoma, predominantly invasive with some non mucinous lepidic component

Invasive mucinous adenocarcinoma (formerly mucinous BAC)

modifications of the classification have implications for surgeons. This relates to sublobar resection for early stage cancer, specific surgical approach for these lesions, extent of lymph node dissection and the role of frozen section analysis. However, definite guidelines cannot be provided yet (2).

For invasive adenocarcinomas, which represent more than 70 to $90 \%$ of surgically resected lung cases, one of the most important aspects of this classification is to present a practical way to address these tumours that are composed of a complex heterogeneous mixture of histological subtypes. Previously, in the 1999/2004 WHO Classification, the term "mixed subtype"was used. The proposal described here is to present a comprehensive histologic subtyping to assess histologic patterns semiquantitatively in 5\% increments, choosing a single predominant pattern. Semiquantitative recording of the patterns in 5\% increments encourages the observer to identify all patterns that may be present, rather than focusing on a single pattern. Individual tumours are then classified according to the predominant pattern and the percentages of the subtypes are also reported. The major histologic patterns are: lepidic (proliferation of type II pneumocytes and Clara cells along the surface alveolar walls), acinar (round to oval-shaped malignant gland invading a fibrous stroma), papillary (malignant cuboidal to columnar tumour cells growing on the surface of fibrovascular cores), micropapillary (small papillary clusters of glandular cells growing within the air spaces, most of which do not show fibrovascular cores), and solid (sheets of tumour cells with abundant cytoplasm and mostly vesicular nuclei with several conspicuous nucleoli) (Figure 1).

An advantage of using semiquantitative comprehensive histologic subtyping is to distinguish multiple primary tumours from intrapulmonary metastases. Indeed, it permits a more precise comparison of multiple tumours. This distinction has a great impact on staging.

Another type of formerly known BAC is the lepidicpredominant adenocarcinoma (LPA). The diagnosis of 


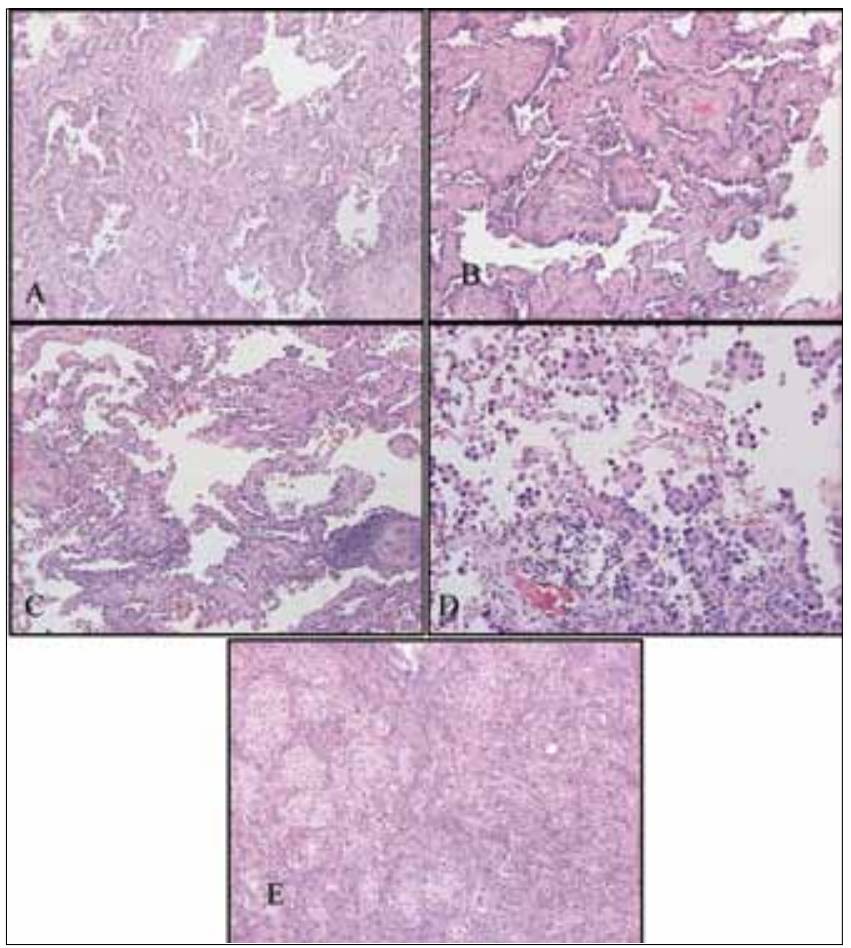

Figure 1. Major histologic pattern of invasive adenocarcinoma A) Acinar adenocarcinoma. B,Papillary adenocarcinoma. C, Lepidic pattern. D) Micropapillary adenocarcinoma. E) Solid adenocarcinoma

LPA rather than MIA is made if the tumour (1) invades lymphatics, blood vessels, or pleura or (2) contains tumour necrosis. For the non-mucinous adenocarcinomas previously classified as mixed subtype where the predominant subtype consists of the former nonmucinous BAC, the term LPA is now recommended.

The micropapillary pattern is especially important for recognizing patients with early-stage disease because it confers a poor prognosis.

Adenocarcinomas, formerly described as mucinous BAC, are currently classified as mucinous AIS, mucinous MIA, or invasive mucinous adenocarcinoma depending on the extent of the lepidic versus invasive growth. They are somewhat different from the rest of lung adenocarcinomas because of the particular immunoprofile (CK20 positive and CK7 negative) and presence of KRAS mutation (combined with virtually absence of EGFR mutation). These characteristics may be confused with lung metastases from the digestive tract.

The interobserver variability in the application of the novel IASLC/ATS/ERS classification was studied by Warth et al. (3). They reviewed 100 archival cases of lung ADC resection specimens. All tumours were classified independently by five pulmonary pathologists and by two residents according to the new clas- sification. The most frequent predominant pattern in this cohort was solid (37\%), followed by acinar (35\%), lepidic (20\%), papillary (5\%) and micropapillary $(3 \%)$ pattern. $\kappa$-values for the denomination of the predominant pattern revealed a substantial agreement for pulmonary pathologists (0.44-0.72) and fair agreement for residents $(0.38-0.47)$. Interobserver variability was significantly higher in cases with higher slide numbers $(p=0.028)$ and was considerably reduced after the initiation of a training session. Intraobserver variability was low ( $\kappa=0.79-0.87)$. Papillary and micropapillary patterns were the most complicated patterns to evaluate, while evaluation of lepidic and solid tumour growth was straightforward. So, these data imply that the novel classification of pulmonary ADC is applicable with acceptable interobserver variability if performed by specifically trained pathologists.

Several authors have demonstrated that this new classification has a prognostic significance. This was shown by Yoshizawa et al. (4) in a series of stage I cases and also by Russel et al. (5) in a series of stages I, II and III adenocarcinomas. Yoshizawa et al. (4) retrospectively reviewed 514 patients who had pathological stage I adenocarcinoma of the lung classified according to the $7^{\text {th }}$ TNM edition, and who had undergone a lobectomy with mediastinal lymph node dissection (6). Comprehensive histological subtyping was used to estimate the percentage of each histological subtype and to identify the predominant subtype. Three overall prognostic groups were identified: low grade: AIS $(n=1)$ and MIA $(n=8)$ had 100\% 5-year disease-free survival; intermediate grade: non-mucinous lepidic predominant $(n=29)$, papillary predominant $(n=143)$ and acinar predominant $(n=232)$ with 90,83 and $84 \%$ 5-year disease-free survival, respectively; and high grade: invasive mucinous adenocarcinoma $(n=13)$, colloid predominant $(n=9)$, solid predominant $(n=67)$ and micropapillary predominant $(n=12)$, with 75,71 , 70 and $67 \%, 5$-year disease-free survival, respectively $(p<0.001)$. Multivariate analysis showed that the different groups of the IASLC/ATS/ERS histological classification $(p=0.038)$ were significant prognostic factors. Maybe in the future, patients with high-grade lesions, even in stage I, will be candidates for adjuvant therapy. Russel et al. (5) investigated the relationship between the new classification and patient survival in a retrospective series of 210 Australian patients with stages I, II, and III lung adenocarcinoma. They confirmed that the new subtypes of AIS, MIA and lepidic-predominant adenocarcinoma had a 5-year survival rate approaching 100\%, whereas micropapillary-predominant and solid with mucin-predominant adenocarcinomas were 
associated with particularly poor survival. Papillarypredominant and acinar-predominant adenocarcinomas had an intermediate prognosis. This effect persisted after controlling for stage. The prognostic impact of the new classification was recently confimed by Warth et al. (7) in a large cohort of 500 patients. They retrospectively studied the architectural pattern of patients with resected ADCs (stages I to IV) They showed that the architectural grading of pulmonary ADCs according to the novel IASLC/ATS/ ERS scheme is a rapid, straightforward, and efficient discriminator for patient prognosis and may support patient stratification for adjuvant chemoradiotherapy. It should be part of an integrated clinical, morphologic, and molecular subtyping to further improve ADC treatment.

The other part of the work of the group of experts from IASLC/ATS/ERS (1) is the establishment of a classification of non-small cell lung carcinomas (NSCLC) for small biopsies and cytology. In fact, the majority of patients with lung cancer $(70 \%)$ will have a diagnosis based on small specimens only, because the disease is generally locally advanced or metastatic at the time of the diagnosis. However the 1999/2004 WHO classifications are based on the histologic examination of surgical specimens, and subclassification in small biopsies is more problematic as such specimens do not sample the entire tumour. In the past, there was no clinical demand for subclassifying NSCLC as this had no specific impact on therapy. However, at the present time, subclassification of NSCLC has significant treatment implications (8). One of the most important steps in the management of NSCLC patient is the distinction between adenocarcinoma and squamous cell carcinoma because, in the new area of targeted therapies, at present only patients with adenocarcinoma are eligible for tyrosine kinase inhibitor (TKI) therapy (if the tumour presents an EGFR mutation), or a pemetrexed or bevacizumab-based chemotherapy regimen. So NSCLC cases have to be further classified into a more specific subtype. There is however universal agreement that most NSCLC can be classified on H\&E (haematoxylin-eosin) stained slides alone without using any immunohistochemical stains. Tumours that are readily classifiable on H\&E-stained slides should not be stained with immunohistochemical markers because of the obligation for the pathologist to keep tissue for molecular testing (EGFR or EML4ALK) (9). However, some NSCLC are too poorly differentiated to be accurately subclassified by standard H\&E criteria, especially in small biopsies. Some markers, especially when used in combination, are indeed useful and accurate in subclassifying poorly differentiated NSCLC. The best panel varies between two (p63/ TTF-1) and four (TTF-1, napsin A, p63, CK5/6) antibodies (10-13).

Strategic use of small biopsy and cytology samples is important in order to preserve as much tissue as possible for potential molecular studies. One way to attain this is for each institution to develop a multidisciplinary team that coordinates the optimal approach to obtain and process biopsy/cytology specimens to provide expeditious diagnostic and molecular results.

In conclusion, the new lung adenocarcinoma classification will substantially modify daily practice but will also improve patient management.

\section{REFERENCES}

1. Travis WD, Brambilla E, Noguchi M, et al. International association for the study of lung cancer/American Thoracic Society/European Respiratory Society international multidisciplinary classification of lung adenocarcinoma. J Thorac Oncol 2011;6:244-85. [CrossRef]

2. Van Schil PE, Asamura H, Rusch VW, et al. Surgical implications of the new IASLC/ATS/ERS adenocarcinoma classification. Eur Respir J 2012;39:478-86. [CrossRef]

3. Warth A, Stenzinger A, von Brünneck AC, et al. Interobserver variability in the application of the novel iaslc/ats/ ers classification. Eur Respir J 2012 Mar 9. [CrossRef]

4. Yoshizawa A, Motoi N, Riely GJ, et al. Impact of proposed IASLC/ATS/ERS classification of lung adenocarcinoma: prognostic subgroups and implications for further revision of staging based on analysis of 514 stage I cases. Mod Pathol 2011;24:653-64. [CrossRef]

5. Russell PA, Wainer Z, Wright GM, et al. Does lung adenocarcinoma subtype predict patient survival?: A clinicopathologic study based on the new International Association for the Study of Lung Cancer/American Thoracic Society/European Respiratory Society international multidisciplinary lung adenocarcinoma classification.J Thorac Oncol 2011;6:1496-504. [CrossRef]

6. Goldstraw P, Crowley J, Chansky K, et al. 7th TNM edition: The IASLC Lung Cancer Staging Project: proposals for the revision of the TNM stage groupings in the forthcoming (seventh) edition of the TNM Classification of malignant tumours. J Thorac Oncol 2007;2:706-14. [CrossRef]

7. Warth A, Muley T, Meister M, et al. The novel histologic International Association for the Study of Lung Cancer/ American Thoracic Society/European Respiratory Society classification system of lung adenocarcinoma is a stage-independent predictor of survival. J Clin Oncol 2012;30:1438-46. [CrossRef]

8. Mukhopadhyay S. Utility of small biopsies for diagnosis of lung nodules: doing more with less. Mod Pathol 2012;25:1054. [CrossRef]

9. Thunnissen E, Kerr KM, Herth FJ, et al The challenge of NSCLC diagnosis and predictive analysis on small sam- 
ples. Practical approach of a working group. Lung Cancer 2012;76:1-18. [CrossRef]

10. Mukhopadhyay S, Katzenstein AL. Subclassification of non-small cell lung carcinomas lacking morphologic differentiation on biopsy specimens: Utility of an immunohistochemical panel containing TTF-1, napsin A, p63, and CK5/6.Am J Surg Pathol 2011;35:15-25. [CrossRef]

11. Loo PS, Thomas SC, Nicolson MC, et al Subtyping of undifferentiated non-small cell carcinomas in bronchial biopsy specimens. J Thorac Oncol 2010;5:442-7. [CrossRef]
12. Rekhtman N, Ang DC, Sima CS, et al Immunohistochemical algorithm for differentiation of lung adenocarcinoma and squamous cell carcinoma based on large series of whole-tissue sections with validation in small specimens. Mod Pathol 2011;24:1348-59. [CrossRef]

13. Righi L, Graziano P, Fornari A, et al Immunohistochemical subtyping of nonsmall cell lung cancer not otherwise specified in fine-needle aspiration cytology: a retrospective study of 103 cases with surgical correlation. Cancer 2011;117:3416-23. [CrossRef] 